\title{
Viewpoint
}

\section{A human skin explant model for predicting graft-versus-host disease following bone marrow transplantation}

\author{
Lisbet Sviland, Anne M Dickinson
}

\begin{abstract}
Graft-versus-host disease (GVHD) is the most serious complication following bone marrow transplantation, with an incidence of $40-60 \%$. The disease can be fatal in $50 \%$ of cases, even in patients receiving marrow from an HLA identical sibling. Several assays have been developed to try to predict the development of GVHD, including mixed lymphocyte culture reaction, cytotoxic $\mathbf{T}$ lymphocyte precursor, and helper $T$ lymphocyte precursor frequency assays. This review describes an in vitro skin explant model which has been used since 1988 for both predicting acute GVHD in HLA identical sibling bone marrow transplantation and studying the pathophysiology of the disease. The model involves sensitising donor lymphocytes in vitro in a primary mixed lymphocyte reaction and then evaluating the secondary response on patient skin biopsies by grading the graft-versus-host reactivity (grades I-IV) histopathologically. From analysis of collective data the model is a clear predictor of GVHD and superior to the other assays widely used, with a correlation of $82 \%$ with clinical outcome. The skin explant model allows the investigator to study the pathogenesis of GVHD. The cytokines TNF $\alpha$ and IFN $\gamma$ are shown to be important mediators of cellular damage in graft-versus-host reactions. Recent work has also involved using the model to study the alloreactivity of cord blood. The model is currently being assessed in several bone marrow transplantation centres in Europe on different patient groups including those who receive marrow from haploidentical and matched unrelated donors.

(F Clin Pathol 1999;52:910-913)
\end{abstract}

Keywords: bone marrow transplantation; graft-versus-host disease; skin explant model

Bone marrow transplantation is increasingly used worldwide to treat a variety of malignant and non-malignant diseases. As the toxicity of the conditioning regimen diminishes and the treatment of infections improves, graft-versus- host disease (GVHD) emerges as the most serious complication of allogeneic grafting. Acute GVHD occurs in 40-60\% of transplants and may be fatal in half of these, even in patients receiving HLA identical sibling or matched unrelated (MUD) donor transplants. ${ }^{1}$ The disease is difficult to treat and several assays have been developed to try to predict its development so that it can be prevented by increased prophylactic immunosuppression. The traditional method widely used as a functional test for alloreactivity between patient and donor is mixed lymphocyte culture (MLC). However, this is unable to determine either the number or the functional capacity of responding $\mathrm{T}$ lymphocytes and has proved insufficiently sensitive to predict the incidence and severity of GVHD after HLA identical sibling, haploidentical, or unrelated donor bone marrow transplants. In recent years, limiting dilution assays of cytotoxic $\mathrm{T}$ lymphocyte precursor (CTLp) ${ }^{2}$ and helper T lymphocyte precursor (HTLp) frequency assays ${ }^{3}$ have been used to detect $\mathrm{T}$ cell alloreactivity of donor against recipient, which cannot be deduced from HLA typing alone. These assays have been carried out either separately or combined and have been used for the detection of alloreactivity between matched unrelated and related transplants. HTLp assays in particular have been shown to be capable of detecting minor histocompatibility antigen (mHag) disparities between HLA identical siblings. ${ }^{4}$

In our laboratory we have used an in vitro skin explant model, originally described by Vogelsang et $a l_{,}^{5}$ since 1988 for both predicting acute GVHD in HLA identical sibling bone marrow transplants ${ }^{6}$ and studying the pathophysiology of the disease. ${ }^{7}$ Analysis of collective data from 56 patients shows that the skin explant model was a clear predictor of GVHD occurrence in 45 of 56 cases $(p<0.0001)$ in HLA and DR matched non-T-cell depleted transplants. ${ }^{89}$ In a recent comparison of the skin explant model with HTLp and CTLp frequency analysis, the human skin explant model remained the more accurate indicator of acute GVHD, with a correlation of $82 \%$ with clinical outcome. ${ }^{8}$ In a preliminary study, Hromadníková et al have used the model to predict 
Table 1 Grading of cutaneous graft-versus-host reaction after Lerner et al ${ }^{12}$

\begin{tabular}{ll}
\hline Grade 0 & Normal skin \\
\hline Grade I & Vacuolisation of epidermal basal cells \\
Grade II & Diffuse vacuolisation of basal cells with dyskeratotic bodies \\
Grade III & Subepidermal cleft formation \\
Grade IV & Complete epidermal separation \\
\hline
\end{tabular}

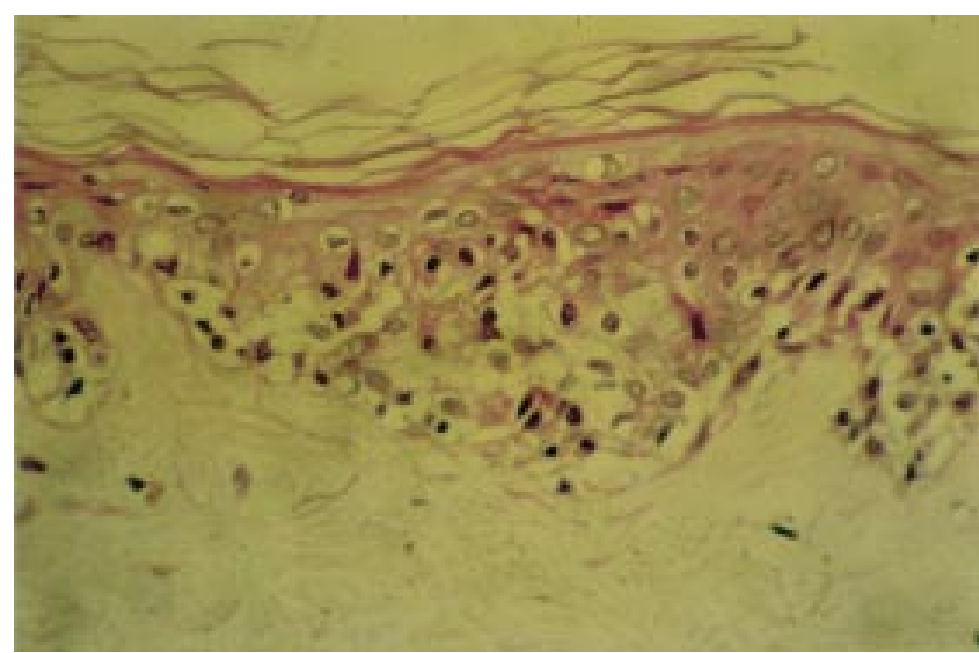

Figure 1 GVHD grade II changes. Skin showing extensive vacuolisation of basal keratinocytes and many scattered dyskeratotic cells in the epidermis.

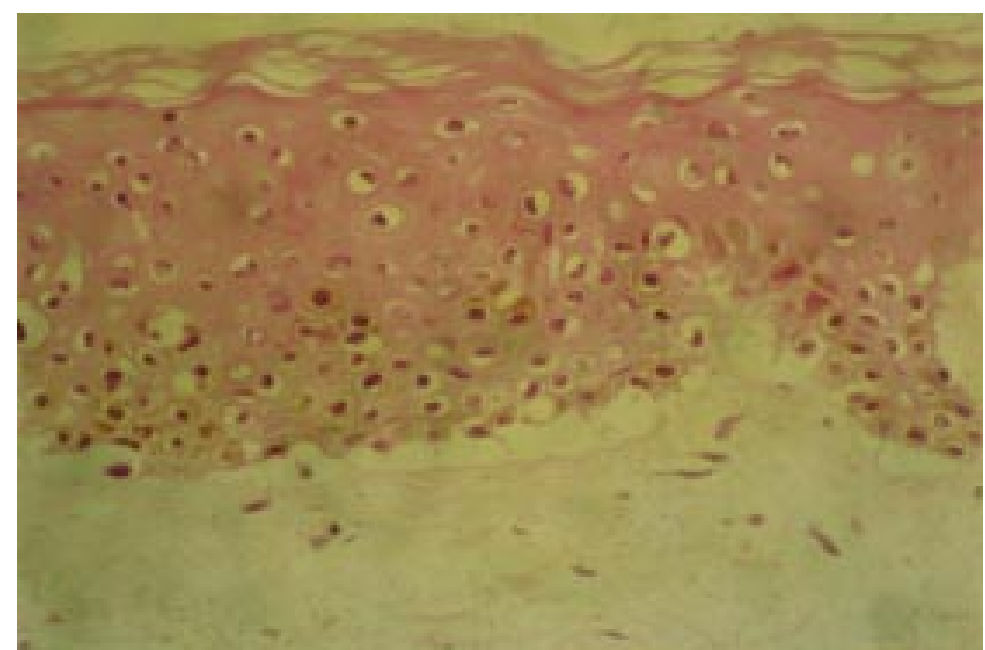

Figure 2 GVHD grade III changes. Confluent vacuolar damage to basal keratinocytes causing cleft formation between the epidermis and dermis.

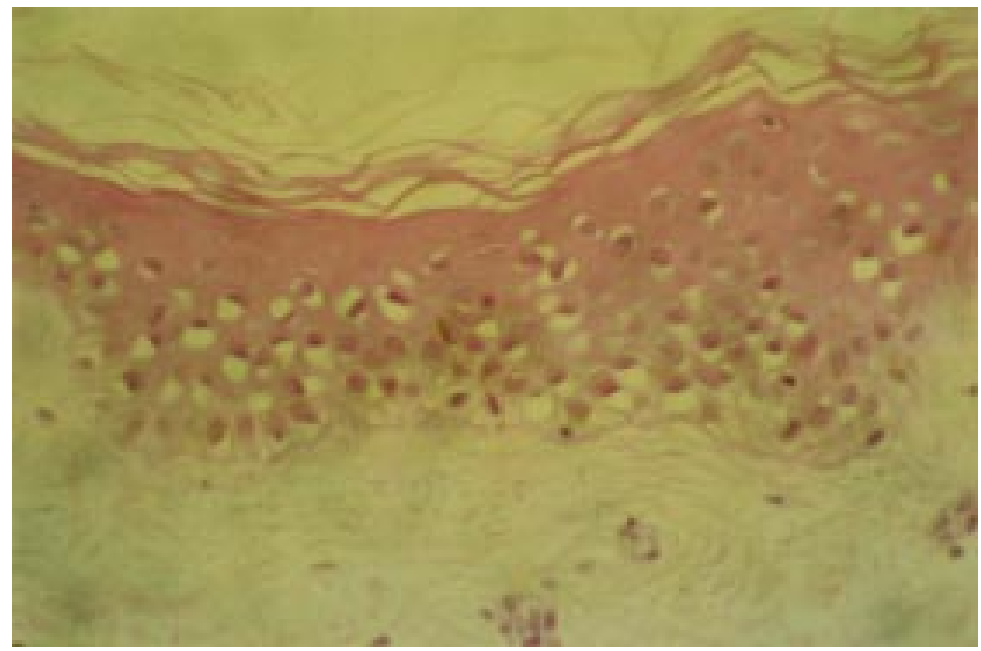

Figure 3 GVHD grade I changes. Skin showing only minor vacuolisation of keratinocytes. acute GVHD in a cohort of paediatric patients in Prague and their results showed a predictive value of $91 \% .^{10}$

\section{The skin explant model}

The Northern Region of England is the only area in Europe which uses the skin explant model routinely for predicting GVHD in HLA and DR compatible bone marrow transplant recipients. ${ }^{11}$ The model is used in our region both as a research tool and to guide the management of individual patients. Briefly, mixed lymphocytes cultures (MLC) consisting of responder lymphocytes from HLA identical donors at a concentration of $5 \times 10^{6}-1 \times 10^{7}$ cells $/ \mathrm{ml}$ are cultured with an equal number of irradiated (20 GY) lymphocytes from the transplant patient (recipient) in $6-10 \mathrm{ml}$ of RPMI-AB (Roswell Park Memorial Institute medium) in $30 \mathrm{ml}$ tissue culture flasks at $37^{\circ} \mathrm{C}$ in $5 \% \mathrm{CO}_{2} / 95 \%$ air. After seven to eight days of culture, responder lymphocytes are washed and resuspended in RPMI 1640 medium supplemented with glutamine, antibiotics, and $10 \%$ heat inactivated autologous serum to the skin biopsy (RPMI-AS); they are then added to patient skin explants at a concentration of $1 \times 10^{6}$ cells/well. The skin biopsies are obtained from the patients following treatment for their primary condition but before conditioning for transplantation.

\section{Histopathological evaluation}

Standard $4 \mathrm{~mm}$ punch biopsies of skin are obtained from the patient. These are trimmed of excess dermis under sterile conditions and divided into 6-10 portions of equal size. Each portion is cultured separately in round bottom microtitre plates with donor lymphocytes as described above. After three days of incubation, explants are fixed in $10 \%$ buffered formalin, sectioned, and stained with haematoxylin and eosin (H\&E). The slides are then assessed for histological evidence of GVHD. Controls are included in every assay and include skin biopsies cultured in medium only and with autologous lymphocytes. The histological findings are not identical to those seen in biopsies taken from patients with clinical GVHD. There are, however, many similarities, especially as the damage seen in the positive biopsies affects the basal layer of the epidermis. The grading system used to evaluate the findings was therefore adapted from that originally described by Lerner et al to diagnose GVHD (table 1) ${ }^{12}$ and extended from grade 0 or 1 to the more severe grade IV.

Only grade II changes or above (figs 1 and 2) are considered important and regarded as positive results. As grade I changes (fig 3 ) are observed when skin biopsies are cultured with autologous lymphocytes or medium alone they are therefore considered as background.

\section{Evaluation of the skin explant model}

The only way to assess the predictive value of the skin explant model is to correlate the histological findings with the clinical history of the patient after transplantation. In their initial report, Berkman et al found that GVHD 
changes of grades II -IV were observed in the skin culture assay and these results correlated with the development of clinical GVHD in five of six HLA compatible marrow donor and recipient pairs. ${ }^{13}$ Further studies by Vogelsang et al verified these results and significantly predicted the outcome of GVHD in (27 of 32) patients with HLA identical bone marrow transplants. ${ }^{5}$ In our laboratory we have now collected data since 1988 and studied 56 HLA and DR matched identical sibling transplants.

The skin explant model correlated with GVHD severity and occurrence in 45 of 56 patients $(\mathrm{p}<0.001){ }^{8}$ Using the same assay, a cohort of paediatric patients has recently been studied and the investigators found a predictive value of $91 \%$ in a cohort of 13 patients. ${ }^{10}$ The assay therefore clearly predicts GVHD. So far only HLA identical sibling transplants have been reported, but there are now centres in Europe taking part in a prospective study to assess the value of the model as a predictive assay for GVHD in matched unrelated transplants as well.

The skin explant model as a research tool Many aspects of GVHD are still poorly understood. The disease clearly involves donor $\mathrm{T}$ lymphocytes, as $\mathrm{T}$ cell depletion of the donor marrow significantly reduces the incidence and severity of clinical GVHD. However, this approach is associated with a high incidence of disease relapse. The current aim therefore is to be able to control a certain amount of graft-versus-host reaction and preserve the graft-versus-leukaemia effect. The skin explant model has been used to study some of the immunological mechanisms that may be associated with the tissue damage seen. In an early study (1988) the role of effector $\mathrm{T}$ cells was investigated by comparing results before and after removal of $\mathrm{CD} 3$ positive, $\mathrm{CD} 4$ positive, and CD8 positive $\mathrm{T}$ cell subsets by antibody and complement cytolysis from responder populations. ${ }^{11}$ Only total removal of CD3 positive $\mathrm{T}$ cells prevented all histopathological changes of GVHD in the skin biopsy specimens. The results also proved that the CD4 positive population caused the greatest degree of GVHD in vitro in the skin explants and that direct infiltration into skin is not required for changes to become evident. Following on from this study, our group investigated cytokine production by sensitised CD 4 and CD 8 enriched populations used in the skin explant model. ${ }^{8}$ This showed that sensitised CD4 populations produced higher tumour necrosis factor $\alpha$ $(\mathrm{TNF} \alpha)$ and $\gamma$ interferon (IFN $\gamma$ ) than sensitised CD8 enriched populations. Cell-free supernatants from these experiments also induced similar histopathological changes in the skin. ${ }^{14}$ The greatest degree of correlation between the GVHD changes observed with responder cells and the supernatant was shown with CD4 enriched MLC. The supernatants were also assayed for TNF $\alpha$ and IFN $\gamma$; CD 4 enriched MLC populations produced high levels of these cytokines. ${ }^{14}$ These results correlated with the grade of GVHD observed in skin explant assays and suggest that $\mathrm{TNF} \alpha$ and
IFN $\gamma$ are directly involved in tissue damage during GVHD. One possible explanation for the observation that CD4 responder cells tend to give rise to the greatest degree of GVHD in an in vitro skin explant assay is that during a mixed lymphocyte reaction they secrete high levels of IFN $\gamma$, which may act synergistically with $\mathrm{TNF} \alpha$ to increase the degree of GVHD observed. Cellular damage caused by supernatant alone could be blocked by preincubation of the supernatant with either anti-TNF $\alpha$ or anti-IFN $\gamma$. The results suggest a role for both these cytokines during GVHD and further suggest that they could act either independently or synergistically to cause cellular damage.

As a negative GVHD result occurred in a proportion of the skin biopsies even in the presence of high levels of TNF $\alpha$ and IFN $\gamma$, this suggests that cytokine receptors need to be present on cells within the skin biopsy section in order for cytokine induced GVHD to be observed; and further that these receptors may need to be induced by responder cells or other cytokines produced by responder cells. The results using CD8 enriched MLC supernatants also suggested that low levels of biologically active cytokines acting synergistically may be sufficient to produce pathogenic effects. It is now known that cytokines other than $\mathrm{TNF} \alpha$ and IFN $\gamma$ are also involved in GVHD. ${ }^{15}$ The stimulation of peripheral blood mononuclear cells with other cytokines such as interleukin (IL) -2 can lead to increased TNF $\alpha$ and IFN $\gamma$ production in vitro ${ }^{16}$ and in vivo. ${ }^{17}{ }^{18}$ It has also been suggested that the cytokine produced and involved in GVHD may depend on the subsets of $T$ cells that are stimulated and are at the site of the lesion. ${ }^{19}$ Clinical studies have shown that low levels of IL-10 production in vitro correlate with increased incidence of GVHD. ${ }^{20}$ High spontaneous levels of IL-10 production before conditioning are associated with lack of TNF $\alpha$ release and the absence of acute GVHD or other severe transplant related complications. ${ }^{21}$ Recent work in our laboratory on cytokine gene polymorphism has shown that certain microsatellites of the IL-10 promoter region are associated with more severe GVHD and this correlates with severity of histopathological damage as measured in the skin explant assay. ${ }^{22}$

Recently human umbilical cord blood has been shown to be a rich source of haematopoietic progenitor cells and could substitute for bone marrow in allogeneic stem cell transplantation. ${ }^{23}$ Cord blood transplantation has been successfully used in children, and in these paediatric recipients the incidence of acute GVHD is very low. ${ }^{24}{ }^{25}$ The immunological mechanism underlying this phenomenon is not clear, so a study was set up to investigate the alloreactive capacity of cord blood cells in vitro using the skin explant model. ${ }^{26}$ The results showed, for the first time, that cord blood mononuclear cells are less able to induce skin GVH type alloreactivity in vitro. Some changes typical of a GVHD reaction were still seen, but the grade of histological damage was less than that induced by peripheral blood mononuclear cells. Thus, while cord blood 
lymphocytes may still be capable of producing some GVHD, its severity appears to be reduced. This observation has been further substantiated by recent results from Rubinstein et $a l .{ }^{27}$ These investigators also presented the first clear in vitro demonstration that a proportion of cord blood mononuclear cell samples were "tolerant" to high levels of HLA mismatches (3-6 Ags), as they did not generate high levels of alloreactive cytotoxic $\mathrm{T}$ lymphocytes or severe skin graft-versus-host type alloreactivity. The results provide a cellular basis for the possibility of using HLA mismatched unrelated cord blood cells as transplant material.

\section{Conclusions}

We have used a skin explant model in various studies to predict GVHD in patients who receive transplants from HLA identical siblings. From analyses of collective data the model is a clear predictor of GVHD and superior to the other assays that are in widespread use, such as the MLC, CTLp, and HTLp assays. The model can be used to aid donor selection where more than one sibling or matched unrelated donor is available. The importance of models such as this lies in the insight they provide into the functional capacity of the alloreactive cells. The skin explant model allows the investigator to study the mechanisms behind the cellular damage associated with GVHD, in particular the role of cytokines. These studies can be extended to investigate adhesion molecules and transduction pathways, apoptosis mechanisms, and cytokine production in relation to cellular damage. The model can also be used to test the role of immunosuppressive drugs in downregulating alloreactive responses, and the results from the GVHD predictive assay may also form the basis of a clinically useful "risk index" for predicting GVHD in HLA identical sibling transplants.

The skin explant model is currently being assessed in several other European centres on different patient groups who receive marrow from haploidentical and matched unrelated donors, where it may in the future prove useful in predicting not just acute but also chronic GVHD, which is more prevalent in these types of transplant.

We wish to thank the Tyneside Leukaemia Research Association, The North of England Cancer Research Campaign, and the European Commission (contract No BIO 4-CT98-0236) for financial support. We are indebted to the clinical bone marrow transplant team in the Department of Haematology, Royal row transplant team in the Department of Haematology, Royal of the research.

1 Ringden O, Deeg HJ. Clinical spectrum of graft-versus-host disease. In: Ferrara JLM, Deeg HJ, Burakoff SJ eds. Graftdisease. In: Ferrara JLM, Deeg HJ, Burakoff SJ eds. Graft-
versus-host disease. New York: Marcel Dekker, 1997:52559.

2 Kaminski E, Hows J, Man S, et al. Prediction of graft versus host disease by frequency analysis of cytotoxic T cells after unrelated bone marrow transplantation. Transplantation 1989;48:608-13

3 Theobold M, Nierle T, Bunjes D, et al. Host specific interleukin-2-secreting donor T-cell precursors as predictors of acute graft-versus-host disease in bone marrow
transplantation between HLA-identical siblings. $N$ Engl $\mathcal{F}$ Med 1992;327:1613-17.
4 Lahance S, Gouvello SL, Roudot F, et al. Predictive value of host specific donor helper $\mathrm{T}$ cell precursor frequency for acute graft versus host disease and relapse in HLA identical siblings receiving allogeneic bone marrow transplantation for hematological malignancies. Transplantation 1997;64: 1147-52.

5 Vogelsang GB, Hess AD, Berkman AW, et al. An in vitro predictive test for graft-versus-host disease in patients with genotypic HLA-identical bone marrow transplants. N Engl $\mathcal{F} \mathrm{Med}$ 1985;313:645-50.

6 Sviland L, Dickinson AM, Carey PJ, et al. An in vitro predictive test for clinical graft versus host disease in allogeneic bone marrow transplant recipients. Bone Marrow Transplant 1990;5:105-9.

7 Dickinson AM, Sviland L, Jackson GH, et al. Monoclonal anti-TNFalpha suppresses graft-versus-host reactions in an in vitro human skin model. Cytokine 1994;6:141-6.

8 Dickinson AM, Sviland L, Jackson GH, et al. Cytokine involvement in predicting clinical graft-versus-host disease (GVHD) in allogeneic bone marrow transplant recipients. Bone Marrow Transplant 1994;13:65-70.

9 Dickinson AM, Sviland L, Wang XN, et al. Predicting graftversus-host disease in HLA-identical bone marrow transplants. A comparison of T-cell frequency analysis and a human skin explant model. Transplantation 1998;66: 857-63

10 Hrodmadnikova I, Sedlacek P, Cermakova M, et al. Predicting graft-versus-host disease using a human skin explant assay in paediatric allogeneic haemopoietic stem cell transplantation. Bone Marrow Transplant (in press).

11 Dickinson AM, Sviland L, Carey $\mathrm{P}$, et al. Skin explant culture as a model for cutaneous graft-versus-host disease in humans. Bone Marrow Transplant 1988;3:323-9.

12 Lerner KG, Kao FG, Storb R, et al. Histopathology of graftversus-host reaction (GVHR) in human recipients of marrow from HLA-matched sibling donors. Transplant Proc 1974;6:367-71.

13 Berkman A, Farmer E, Tutschka P, et al. Skin explant culture as a model for cutaneous graft versus host disease. Exp Hematol 1982;10(suppl 12):33-40.

14 Dickinson AM, Sviland L, Dunn J, et al. Demonstration of direct involvement of cytokines in graft-versus-host reactions using an in vitro human skin explant model. Bone Marrow Transplant 1991;7:209-16.

15 Lichtman AH, Krenger W, Ferrara JLM. Cytokines networks. In: Ferrara JLM, Deeg HJ, Burakoff SJ, eds. Graft versus host disease. New York: Marcel Dekker, 1997:179218.

16 Limb GA, Meager A, Wolley J, et al. Release of cytokine during generation of lymphokine activated killer (LAK) cells by IL-2. F Immunol 1989;68:514-19.

17 Heslop HE, Gottlieb DJ, Reittie JE, et al. Spontaneous and interleukin-2 induced secretion of tumor necrosis factor and gamma interferon following autologous marrow transplantation in chemotherapy. Br Ұ Haematol 1989;72:122-6.

18 Heslop HE, Gottlieb DJ, Bianchi ACM, et al. In vivo induction of gamma interferon and tumor necrosis factor by interleukin-2 infusion following intensive chemotherapy on autologous marrow transplantation. Blood 1989;74: 1374-80.

19 Nakhleh RE, Snover DC, Weisdorf S, et al. Immunopathology of graft versus host disease in the upper gastrointestinal tract. Transplantation 1989;48:61-5.

20 Korholz D, Hempel L, Packeisen J, et al. Significance of interleukin-10 for acute graft- versus-host disease in interleukin-10 for acute graft- versus-host disease in transplantation [abstract]. Klin Padiatr 1996;208:141-4.

21 Holler E, Roncarolo MG, Hintermeier-Knabe R, et al. Low incidence of transplant related complications in patients with high spontaneous IL-10 production prior to conditioning - evidence for a protective role of IL-10 in allogeneic BMT [abstract]. Bone Marrow Transplant 1995;5(S2):S59.

22 Middleton PG, Taylor PRA, Jackson G, et al. Cytokine gene polymorphisms associating with severe acute graft-versushost disease in HLA identical sibling transplants. Blood 1998;92:3943-8.

23 Broxmeyer HE, Douglas GW, Hangoc G, et al. Human umbilical cord blood as a potential source of transplantable hematopoietic stem/progenitor cells. Proc Natl Acad Sci USA 1989;86:3828-32.

24 Wagner JE, Kernan NA, Steinbuch M, et al. Allogeneic sibling umbilical cord blood transplantation in children with malignant and non-malignant disease. Lancet 1995;346: 214-19.

25 Kurzberg J, Laughlin M, Graham M, et al. Placental blood as a source of haematopoeitic stem cells for transplantation into unrelated recipients. N Engl F Med 1996;335:157-66.

26 Wang XN, Sviland L, Ademokun AJ, et al. Cellular alloreactivity of human cord blood cells detected by T-cell tivity of human cord blood cells detected by T-cell
frequency analysis and a human skin explant model. Transfrequency analysis and a hum
plantation 1998;66:903-9.

27 Rubinstein P, Carrier C, Scaradovou A, et al. Outcome among 562 recipients of placental-blood transplants from unrelated donors. N Engl f Med 1998;339:1565-77. 